\title{
Propriedades físicas e reológicas de produtos à base de frutas
}

Physical and rheological properties of fruit products

\section{Sílvia Cristina Sobottka Rolim de Moura ${ }^{1 *}$, Fernanda Zaratini Vissotto², Cristiane Rodrigues Gomes Ruffi ${ }^{2}$, Paulo Alves Júnior ${ }^{3,4}$}

${ }^{1}$ Instituto de Tecnologia de Alimentos (ITAL), Fruthotec, Campinas/SP - Brasil

2Instituto de Tecnologia de Alimentos (ITAL), Cereal Chocotec, Campinas/SP - Brasil

${ }^{3}$ Universidade Estadual de Campinas (UNICAMP), Faculdade de Engenharia de Alimentos (FEA), Campinas/SP - Brasil

${ }^{4}$ Conselho Nacional de Desenvolvimento Científico e Tecnológico - CNPQ, Campinas/SP - Brasil

\section{*Corresponding Author}

Sílvia Cristina Sobottka Rolim de Moura, Instituto de Tecnologia de Alimentos (ITAL), Fruthotec, Av. Brasil, 2880, Jd. Chapadão, CEP: 13070-178, Campinas/SP - Brasil, e-mail: smoura@ital.sp.gov.br

Cite as: Physical and rheological properties of fruit products. Braz. J. Food Technol., v. 19, e2015086, 2016.

Received: Nov. 06, 2015; Accepted: Aug. 11, 2016

\section{Resumo}

Em vista da crescente demanda da população por questões relacionadas à saúde, nutrição e bem-estar, o mercado alimentício tem se preocupado em aumentar a inserção de ingredientes mais saudáveis como as frutas, por exemplo, visando atender à nova demanda do consumidor. A otimização de um processo é dependente da presença de dados precisos que permitam o dimensionamento correto dos equipamentos. O objetivo deste trabalho foi caracterizar amostras comerciais de produtos à base de frutas e avaliar as propriedades termofísicas (densidade, calor específico, difusividade térmica e condutividade térmica) e reológicas (viscosidade aparente e energia de ativação), visando à adequação a modelos teóricos. Os resultados foram ajustados a modelos matemáticos apresentando erros relativos entre 0,2\% e 50\%, sendo o modelo de ajuste da densidade o mais preciso (erro máximo de 1,7\%). O comportamento reológico das amostras teve melhor ajuste ao modelo Lei da Potência $\left(R^{2}>0,96\right)$ e a viscosidade relacionou-se bem com a temperatura, segundo a equação de Arrhenius $\left(R^{2}>0,90\right)$.

Palavras-chave: Preparados de fruta; Reologia; Propriedades térmicas; Caracterização física.

\section{Summary}

In view of the increasing demand of the population with respect to health-related issues, nutrition and wellbeing, the food market has been concerned to increase the inclusion of healthier ingredients such as fruits, for example, to meet this new consumer demand. The optimization of a process is dependent on the presence of accurate data to allow for the correct sizing of the equipment. The aim of this study was to characterize commercial samples of fruit-based products and evaluate their thermophysical (density, specific heat, thermal diffusivity and thermal conductivity) and rheological (viscosity and apparent activation energy) properties in order to fit the theoretical models. The results were adjusted to mathematical models showing relative errors between 0.2 and $50 \%$, the density adjustment model (maximum error of $1.7 \%$ ) being the most accurate. The rheological behavior of the samples fitted the power law model best $\left(R^{2}>0.96\right)$, and the viscosity correlated well with the temperature according to the Arrhenius equation $\left(R^{2}>0.90\right)$.

Keywords: Fruit preparations; Rheology; Thermal properties; Physical characterization. 


\section{Introdução}

Entre as tendências dos consumidores mundiais de alimentos estão as relacionadas a "Saudabilidade e Bem-estar" (BRASIL..., 2010). Essas tendências originaram-se em fatores como o envelhecimento das populações, as descobertas científicas que vinculam determinadas dietas às doenças, bem como à renda e vida nas grandes cidades, influenciando a busca de um estilo de vida mais saudável. São diversos os segmentos que surgem a partir dessas tendências, entre os quais é possível destacar a procura de alimentos funcionais, os produtos para dietas e controle de peso e o crescimento de produtos naturais ou com inserção de ingredientes naturais, como frutas e vegetais (BRASIL..., 2010).

Segundo Zulueta et al. (2007), o uso de bases de frutas como parte da formulação de produtos lácteos e de confeitaria tem apresentado constante crescimento. Normalmente existe necessidade de redimensionamento de equipamentos e de processo e, para tal, faz-se necessário o conhecimento de propriedades e padrões de comportamento dos novos ingredientes (TOLEDO, 2007).

Os preparados à base de frutas são formulados prioritariamente de uma fonte de fruta (polpa, suco concentrado, fruta em pedaços, ou combinações destes), açúcar como forma de veículo e incorporador de sólidos, espessantes e estabilizantes. Com finalidade de padronização de produto também é comum incorporar corantes e aromatizantes (BARRET et al., 2005). Bases e preparados são utilizados pela indústria de sobremesas, com ênfase maior na indústria de laticínios, sorvetes e confeitaria. Estes produtos têm a conveniência de uma padronização específica para o emprego do processo em questão, maior garantia da segurança alimentar, melhor homogeneidade de lotes de produção e melhor controle logístico e de estoque por parte da indústria (ZULUETA et al., 2007).

O dimensionamento dos equipamentos utilizados no processamento de alimentos, principalmente aqueles que trabalham com bombeamento e transferência de calor, exigem dados precisos das propriedades térmicas dos alimentos fluidos e semissólidos (densidade, condutividade térmica, difusividade térmica e calor específico) e de como essas propriedades se comportam durante o processo, em função da temperatura (ROUSTAPOUR; GAZOR, 2013).

As propriedades reológicas de alimentos têm um papel importante durante os processos de desenvolvimento, produção e processamento. Os processos industriais envolvem uso de diversas operações unitárias que se relacionam diretamente com as propriedades reológicas. O transporte de matérias é utilizado durante os diversos estágios de processamento ou na fase de envase por meio de bombeamento, bem como processos de agitação e homogeneização. O conhecimento do comportamento dos fluidos é necessário ao correto dimensionamento de instalações, processos, equipamentos e tubulações (Sharma et al., 2000).

Viscosidade é a propriedade física que caracteriza a resistência de um fluido ao escoamento, a uma dada temperatura. A dependência desta propriedade com a alteração de temperatura varia para cada matéria e, em geral, processos trabalham com diversas fases de temperatura, e o conhecimento da razão entre estas duas grandezas é o que permite manter um regime de processamento contínuo (RAO, 2006).

Fluidos newtonianos não alteram sua viscosidade com a variação da taxa de cisalhamento. Fluidos não newtonianos podem ser classificados conforme a variação de sua viscosidade aparente em função da taxa de deformação: pseudoplásticos tendem a diminuir a viscosidade aparente conforme aumenta a taxa de deformação e os dilatantes tendem a aumentar a viscosidade aparente conforme se aumenta a taxa de deformação (RAO; RIZVI, 1995; DIAMANTE; UMEMOTO, 2015).

As propriedades termofísicas são de suma importância na elaboração, otimização e implementação de projetos na área de engenharia de alimentos. Ainda que existam modelos de predição teórica presentes na literatura, geralmente tomando como base a composição centesimal do alimento, é bastante comum que tais modelos apresentem erros. Assim, em muitos casos, vê-se a necessidade da obtenção de dados de forma prática, bem fundamentados e confiáveis (RAO, 2006).

Em vista do exposto, o objetivo do trabalho foi a obtenção das propriedades termofísicas e reológicas de produtos à base de frutas e a comparação a modelos teóricos e a dados apresentados na literatura.

\section{Material e métodos}

Foram avaliadas cinco amostras distintas de preparados e bases de frutas obtidas no comércio local. Foram elas: duas coberturas para sorvete nos sabores morango e limão (CM e CL), duas bases de frutas nos sabores maracujá e coco (BM e PC) e um smoothie de frutas vermelhas (SM).

As amostras foram caracterizadas quanto a $\mathrm{pH}$, sólidos solúveis, atividade de água e cor instrumental, todas estas realizadas em triplicata, empregando os seguintes métodos:

Análises de $\mathrm{pH}$ de forma direta em potenciômetro (Digimed, modelo DM-20, Brasil) (IAL, 1985); Sólidos solúveis por uso de refratômetro óptico portátil (ATAGO, modelo PAL- $\alpha$, Japão) (IAL, 1985); Análise de atividade de água diretamente em medidor de atividade de água (Aqualab, série 3TE, modelo cx-2, Brasil), na temperatura constante de $25^{\circ} \mathrm{C}$; Análises de cor instrumental diretamente em colorímetro (Minolta, modelo CR-400, Japão), por 
uso das coordenadas retangulares: $L^{*}$ representando a luminosidade, $a^{\star}$ representando o eixo verde-vermelho e b* para a representação do eixo amarelo-azul, conforme sistema CIELAB, criado em 1976 (HILL et al., 1997).

As propriedades termofísicas avaliadas foram: calor específico, difusividade térmica, densidade e condutibilidade térmica. Analisou-se a densidade segundo a metodologia de deslocamento de fluidos em picnômetros, descrita na norma n. ${ }^{\circ} 985.19$ da AOAC (WILLIAMS, 1990), em três diferentes temperaturas, mantidas constantes por uso de banho ultratermostático (Marqlabor, modelo BUT-10, Brasil).

A análise do calor específico foi realizada por método indireto de misturas descrito na literatura por Hwang e Hayakawa (1979) e Budžaki e Šeruga (2015). Utilizou-se um calorímetro de mistura que consiste de uma garrafa térmica de capacidade volumétrica de 1 litro (Figura 1). As amostras do produto (50 a $100 \mathrm{~g}$ ) foram acondicionadas em embalagens plásticas de polietileno e previamente colocadas em geladeira ( $10^{\circ} \mathrm{C}$ por 24 horas), a fim de garantir a estabilização da temperatura.

Adicionou-se ao calorímetro $500 \mathrm{~g}$ a $600 \mathrm{~g}$ de água destilada a $90 \pm 2{ }^{\circ} \mathrm{C}$. Logo após, o calorímetro é disposto em um "shaker". A embalagem plástica contendo a amostra foi introduzida, com o cuidado de se determinar a temperatura inicial no seu interior.

Fez-se o registro contínuo da temperatura no interior do calorímetro a cada 30 segundos com a utilização de um aquisitor de dados (Almeno, MA2890-9, Alemanha). O sistema foi agitado continuamente em um "shaker". A coleta de dados de temperatura foi efetuada até o equilíbrio térmico. A regressão linear foi determinada pela etapa linear da curva de temperatura versus tempo. Para os cálculos do calor específico, empregou-se a Equação 1, que é obtida através de balanço de energia.

(c)

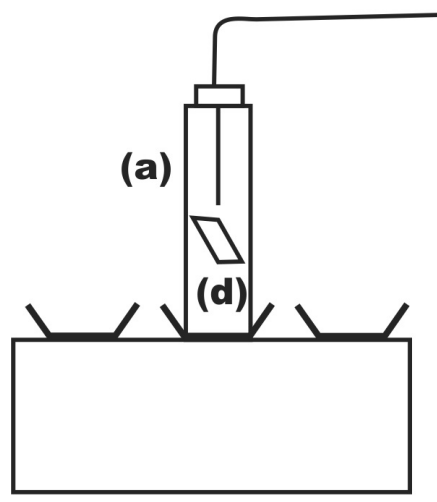

(e)

Figura 1. Esquema do aparato para medição de calor específico: (a) calorímetro; (b) módulo de aquisição de dados; (c) cabo termopar; (d) amostra; (e) agitador.

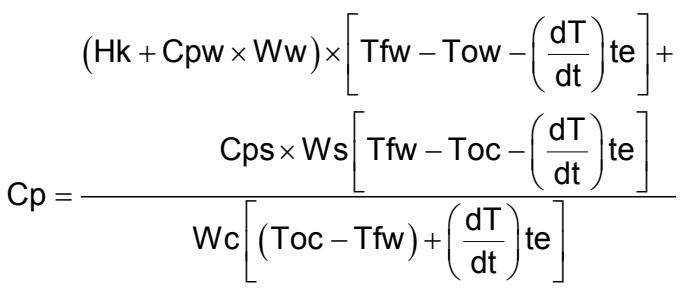

Onde: $\mathrm{Cp}=$ calor específico $\left(\mathrm{kJ} /\left(\mathrm{kg} .{ }^{\circ} \mathrm{C}\right)\right)$ da amostra; $\mathrm{Hk}=$ capacidade térmica do calorímetro $\left(\mathrm{kJ} /\left(\mathrm{kg} .{ }^{\circ} \mathrm{C}\right)\right)$; Cpw = calor específico da água destilada $\left(\mathrm{kJ} /\left(\mathrm{kg} .{ }^{\circ} \mathrm{C}\right)\right)$; $\mathrm{Ww}=$ massa da água destilada $(\mathrm{kg}) ; \mathrm{Tfw}=$ temperatura referente ao início da reta na curva tempo $\times$ temperatura da água $\left({ }^{\circ} \mathrm{C}\right)$; Tow = temperatura inicial da água destilada $\left({ }^{\circ} \mathrm{C}\right) ; \mathrm{dT} / \mathrm{dt}=$ inclinação da curva tempo $\times$ temperatura da água destilada $\left({ }^{\circ} \mathrm{C} / \mathrm{s}\right)$; te $=$ tempo referente a Tfw da curva tempo $\times$ temperatura da água destilada (s); Cps = calor específico da embalagem de polietileno $(\mathrm{kJ} / \mathrm{kg}) ; \mathrm{Ws}=$ massa da embalagem de polietileno (g); Wc = massa da amostra (g); Toc = temperatura inicial da amostra $\left({ }^{\circ} \mathrm{C}\right)$.

O método utilizado na determinação da difusividade térmica foi descrito na literatura por Dickerson (1965) e Barbosa et al. (2013). O sistema consiste de uma célula de aço inox, de 0,05 m de diâmetro e 0,298 m de altura, com dois termopares acoplados, devidamente calibrados - um na superfície e outro, tipo agulha, disposto no plano central da amostra (Figura 2).

O aparato de medição foi então disposto em banho termostático (Eletric Company, modelo Magni Whirl, EUA) de água a $20{ }^{\circ} \mathrm{C}$. Depois do equilíbrio térmico entre a célula e o banho, iniciou-se o aquecimento, com uma taxa de $0,7^{\circ} \mathrm{C} / \mathrm{min}$, até que a temperatura média da amostra atingisse aproximadamente $80^{\circ} \mathrm{C}$. As medidas de temperatura foram registradas em intervalos de 1 minuto, por meio de um aquisitor de dados (Almeno, MA2890-9,

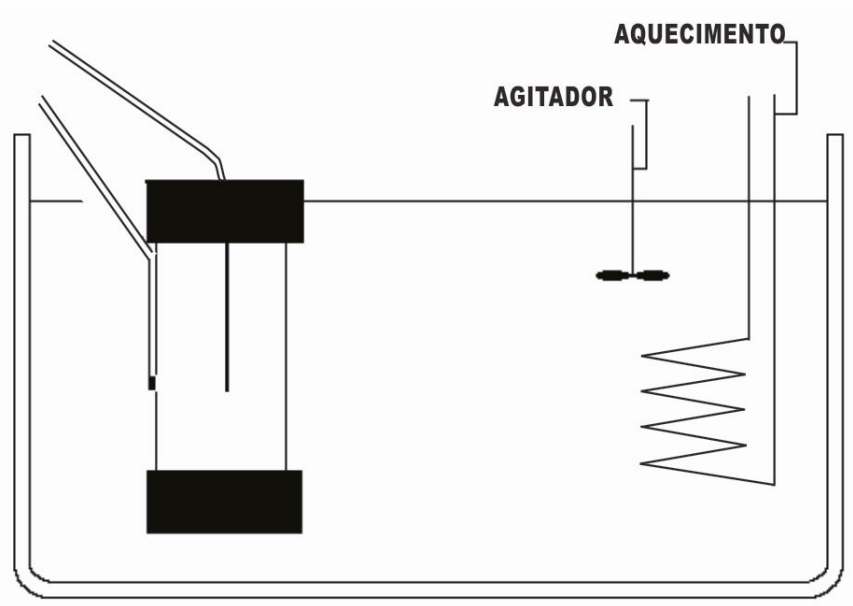

Figura 2. Esquema do aparato para medição de difusividade térmica. 
Alemanha). Os dados obtidos servem de base para o cálculo da difusividade térmica pela Equação 2.

$$
\alpha=\frac{A \times R^{2}}{4 \times\left(T_{\text {ext }}-T_{\text {int }}\right)}
$$

Onde: $\alpha=$ difusividade térmica; $A$ = taxa de subida de temperatura do banho $\left({ }^{\circ} \mathrm{C} / \mathrm{min}\right) ; \mathrm{R}=$ raio da célula $(\mathrm{m})$; $\left(T_{\text {ext }}-T_{\text {int }}\right)=$ diferença entre as temperaturas externa e interna da célula $\left({ }^{\circ} \mathrm{C}\right)$.

O valor calculado para a difusividade foi obtido para cada temperatura, fazendo-se posteriormente a média dos valores na faixa estudada.

A condutividade térmica foi determinada após o conhecimento da densidade $(\rho)$, da difusividade térmica $(\alpha)$ e do calor específico (Cp) do produto, usando a Equação 3.

$\mathrm{k}=\rho \times \mathrm{Cp} \times \alpha$

As amostras foram também avaliadas quanto ao comportamento reológico em reômetro rotacional programável, marca BROOKFIELD, modelo DVIII+, com uso do adaptador de pequenas amostras, "spindle" cilíndrico (especificação S15), acoplado a um banho com controle termostático (Brookfield, TC-500, EUA).

O comportamento reológico de escoamento foi realizado em cinco temperaturas $\left(25,35,50,65\right.$ e $\left.90^{\circ} \mathrm{C}\right)$, ajustando a programação do reômetro para 20 rpm por 120 segundos, para cada temperatura e coletados os dados de viscosidade aparente $(\mu)$. A Equação de Arrhenius (4) foi utilizada para verificar o ajuste dos dados.

$\ln \mu=\ln \mu_{0}+\frac{E_{a}}{R \times T}$
Onde: $\mu_{0}=$ viscosidade aparente inicial; $\mathrm{Ea}=$ energia de ativação (kJ/mol); R = 8,314 (J/(mol.Kelvin)); $T$ = temperatura (Kelvin).

O perfil reológico do fluido foi realizado em três temperaturas, 25,50 e $90^{\circ} \mathrm{C}$ com programação ajustada para variação de taxa de deformação de 0 a $50 \mathrm{~s}^{-1}$. Foram obtidos os resultados de tensão de cisalhamento, para cada taxa de deformação aplicada. Os dados obtidos nos experimentos foram analisados estatisticamente por meio de programas computacionais (STATISTICA, versão 10, StatSoft), ajustando-se aos modelos teóricos de Ostwald-de-Waelle ou Lei da Potência (5) e de Herschel-Bulkley (6).

$$
\begin{aligned}
& \sigma=K(\Upsilon)^{n} \\
& \sigma=\sigma_{0}+\eta\left(\Upsilon^{n}\right)
\end{aligned}
$$

Onde: $\sigma=$ tensão de cisalhamento $(\mathrm{Pa}) ; \Upsilon=$ taxa de deformação $\left(\mathrm{s}^{-1}\right) ; K=$ índice de consistência $\left(P a . s^{n}\right)$; $\mathrm{n}=$ índice de comportamento; $\sigma_{0}=$ tensão de cisalhamento inicial $(\mathrm{Pa}) ; \eta=$ viscosidade plástica (Pa.s).

\section{Resultados e discussão}

A Tabela 1 apresenta os resultados das análises de sólidos solúveis ( ${ }^{\circ}$ Brix), atividade de água (Aw) e pH das amostras.

Os resultados de caracterização mostram que os produtos à base de fruta tendem a manter um pH próximo ao característico da fruta. Os valores de atividade de água e sólidos solúveis ( ${ }^{\circ}$ Brix) são bastante diferentes, indicando assim que os preparados não são padronizados e variam com o tipo de uso destinado e frutas base.

Os resultados das leituras de cor durante o período de 120 dias foram avaliados estatisticamente e demonstraram não haver diferenças significativas $(p \leq 0,05)$ entre as leituras, atestando a boa estabilidade de cor nas amostras avaliadas (Tabela 2).

Tabela 1. Características físico-químicas de produtos à base de frutas.

\begin{tabular}{cccc} 
Amostra & pH & Aw & Sólidos solúveis ( ${ }^{\circ}$ Brix) \\
CL & $2,29 \pm 0,01$ & $0,647 \pm 0,01$ & $83,3 \pm 0,7$ \\
CM & $3,00 \pm 0,01$ & $0,618 \pm 0,01$ & $81,1 \pm 0,9$ \\
BM & $3,30 \pm 0,01$ & $0,950 \pm 0,02$ & $36,3 \pm 0,2$ \\
PC & $5,20 \pm 0,01$ & $0,968 \pm 0,01$ & $29,4 \pm 0,8$ \\
SV & $3,88 \pm 0,01$ & $0,976 \pm 0,02$ & $14,5 \pm 0,1$ \\
\hline
\end{tabular}

Tabela 2. Cor instrumental dos produtos à base de frutas durante 120 dias.

\begin{tabular}{cccc} 
Amostra & $\mathbf{L}^{*} \pm$ desvio (120 dias) & $\mathbf{a}^{*} \pm$ desvio (120 dias) & $\mathbf{b}^{*} \pm$ desvio (120 dias) \\
CL & $25,26 \pm 0,03$ & $-11,15 \pm 0,04$ & $8,85 \pm 0,08$ \\
CM & $24,01 \pm 0,01$ & $13,74 \pm 0,05$ & $4,72 \pm 0,06$ \\
BM & $28,29 \pm 0,07$ & $2,11 \pm 0,03$ & $10,27 \pm 0,06$ \\
PC & $22,02 \pm 0,01$ & $1,23 \pm 0,01$ & $2,01 \pm 0,01$ \\
SM & $29,86 \pm 0,02$ & $11,89 \pm 0,07$ & $1,04 \pm 0,04$ \\
\hline
\end{tabular}

$\mathrm{L}^{*}=$ Luminosidade (0 preto e 100 branco); $\mathrm{a}^{*}$ = coordenada vermelho/verde (+a indica vermelho e -a indica verde); $b^{*}=\mathrm{coordenada}$ amarelo / azul (+b indica amarelo e $-b$ indica azul). 
A Figura 3 apresenta a influência da temperatura na viscosidade aparente das amostras estudadas, mostrando o decréscimo da viscosidade com o aumento da temperatura. Segundo Vidal et al. (2006), um aumento da temperatura diminui a viscosidade da fase líquida, aumentando o movimento das partículas em suspensão e causando o decréscimo da viscosidade do produto. Estas informações são de extrema relevância em processos industriais, especialmente no que tange às operações unitárias de transferência de quantidade de movimento e massa.

Os dados de viscosidade aparente versus temperatura, na taxa de deformação de 20 rpm por 120 segundos, ajustam-se à Equação de Arrhenius (4). Tais dados são representados pela Figura 4 e sumarizados na Tabela 3. Nota-se que os valores da energia de ativação $\left(E_{a}\right)$ foram maiores para as amostras de coberturas e menores para as bases e smoothie.

A energia de ativação é maior quanto menor for a viscosidade aparente $(\mu)$ das amostras. Em geral, quanto maior a energia de ativação, maior é o efeito da temperatura sobre a viscosidade (PEREIRA et al., 2014; DIAMANTE; UMEMOTO, 2015). Os resultados encontrados concordam com a literatura já que as amostras CL e CM apresentaram maiores alterações na viscosidade aparente e, consequentemente, maiores valores energia de ativação.

Foram testados os ajustes às equações de Ostwald-de-Waelle ou Lei da Potência (5) e HerschelBulkley (6).

Em geral, ambos os modelos testados têm boa inserção com os dados obtidos experimentalmente, entretanto, como observação dos tratamentos estatísticos, conclui-se que o modelo da Lei da Potência (5) é o mais indicado, por apresentar os melhores ajustes (maiores valores de $R^{2}$ ).

O modelo de Herschel-Bulkley (6) apresentou ajuste um pouco inferior e, devido às baixas taxas de cisalhamento que provocaram oscilações nas medidas, não foi possível a determinação precisa da tensão inicial $\left(\sigma_{0}\right)$.

A Figura 5 é apresentada como exemplo do ajuste ao modelo Lei da Potência dos resultados de tensão de cisalhamento em função da alteração da taxa de deformação.

A Tabela 4 apresenta os parâmetros do ajuste ao modelo Lei da Potência, nas temperaturas em que foram analisados.

O ajuste ao modelo indicou que para todas as amostras os valores de " $n$ " foram inferiores a 1. Sendo assim, as amostras têm seu comportamento reológico classificado como sendo de um fluido não newtoniano pseudoplástico.

O aumento da temperatura, em todas as amostras analisadas, refletiu numa diminuição do índice de
Tabela 3. Valores de energia de ativação e coeficiente de adequação para o modelo de Arrhenius.

\begin{tabular}{ccc} 
Amostra & $\mathbf{E}_{\mathbf{a}}(\mathbf{k J} / \mathbf{m o l})$ & $\mathbf{R}^{\mathbf{2}}$ \\
CL & 54,4 & 0,91 \\
CM & 52,4 & 0,95 \\
BM & 23,3 & 0,98 \\
PC & 11,20 & 0,96 \\
SV & 16,6 & 0,92 \\
\hline
\end{tabular}

$\mathrm{Ea}=$ energia de ativação.

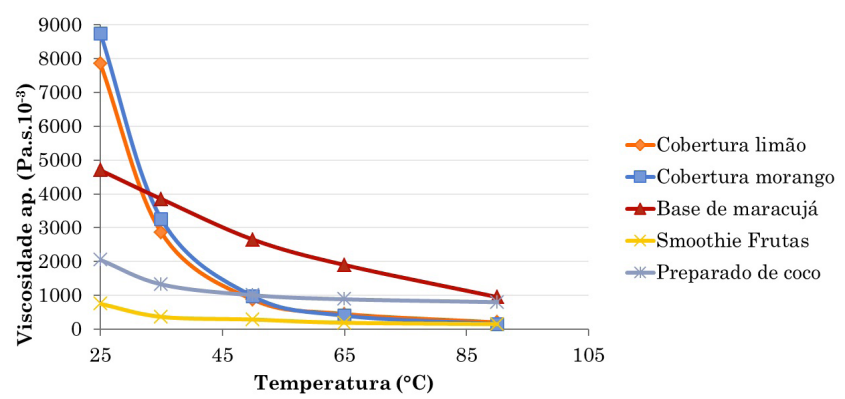

Figura 3. Representação das curvas de viscosidade aparente dos produtos à base de frutas com alteração da temperatura $(\Upsilon=20 \mathrm{rpm}$ por $120 \mathrm{~s})$.

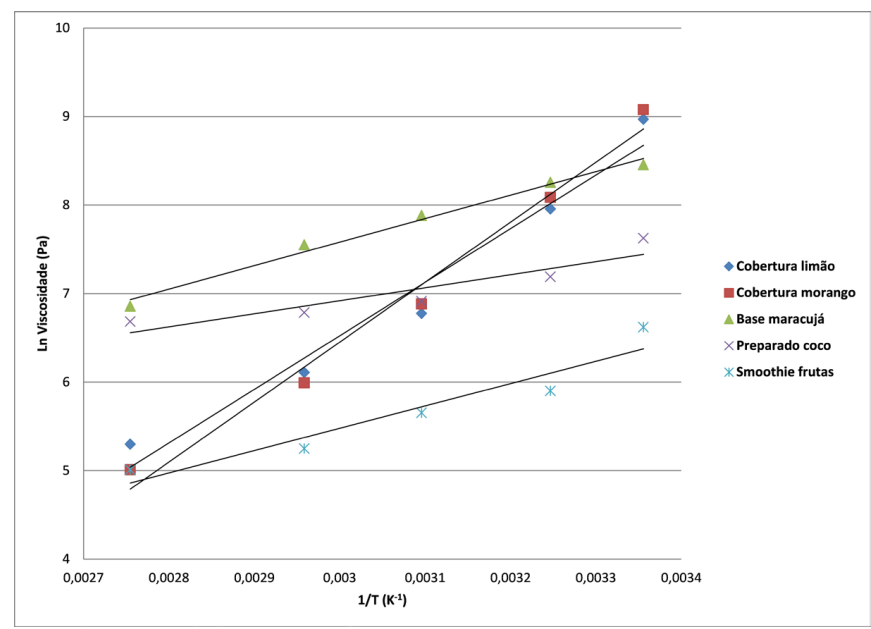

Figura 4. Ajuste ao modelo de Arrhenius.

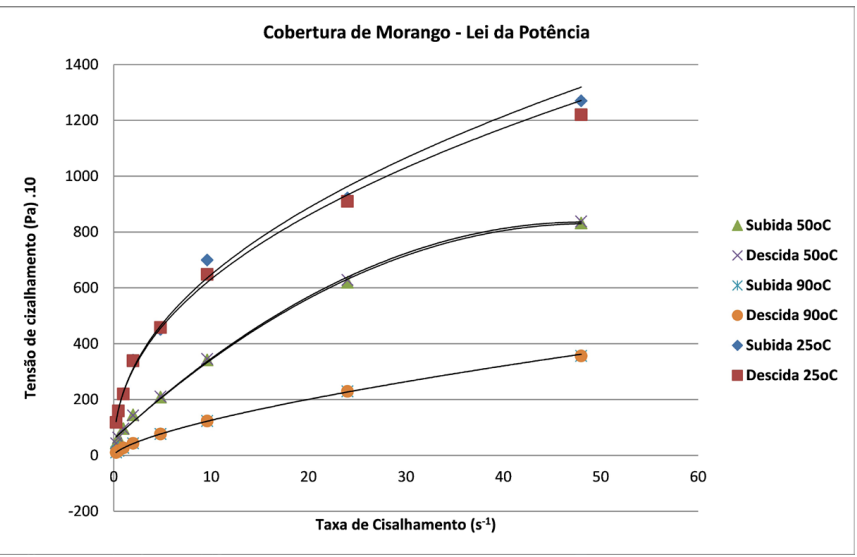

Figura 5. Ajuste ao modelo Lei da Potência para os resultados da amostra de CM. 
Propriedades físicas e reológicas de produtos à base de frutas

Moura, S. C. S. R. et al.

Tabela 4. Ajuste ao modelo da Lei da Potência para as amostras avaliadas.

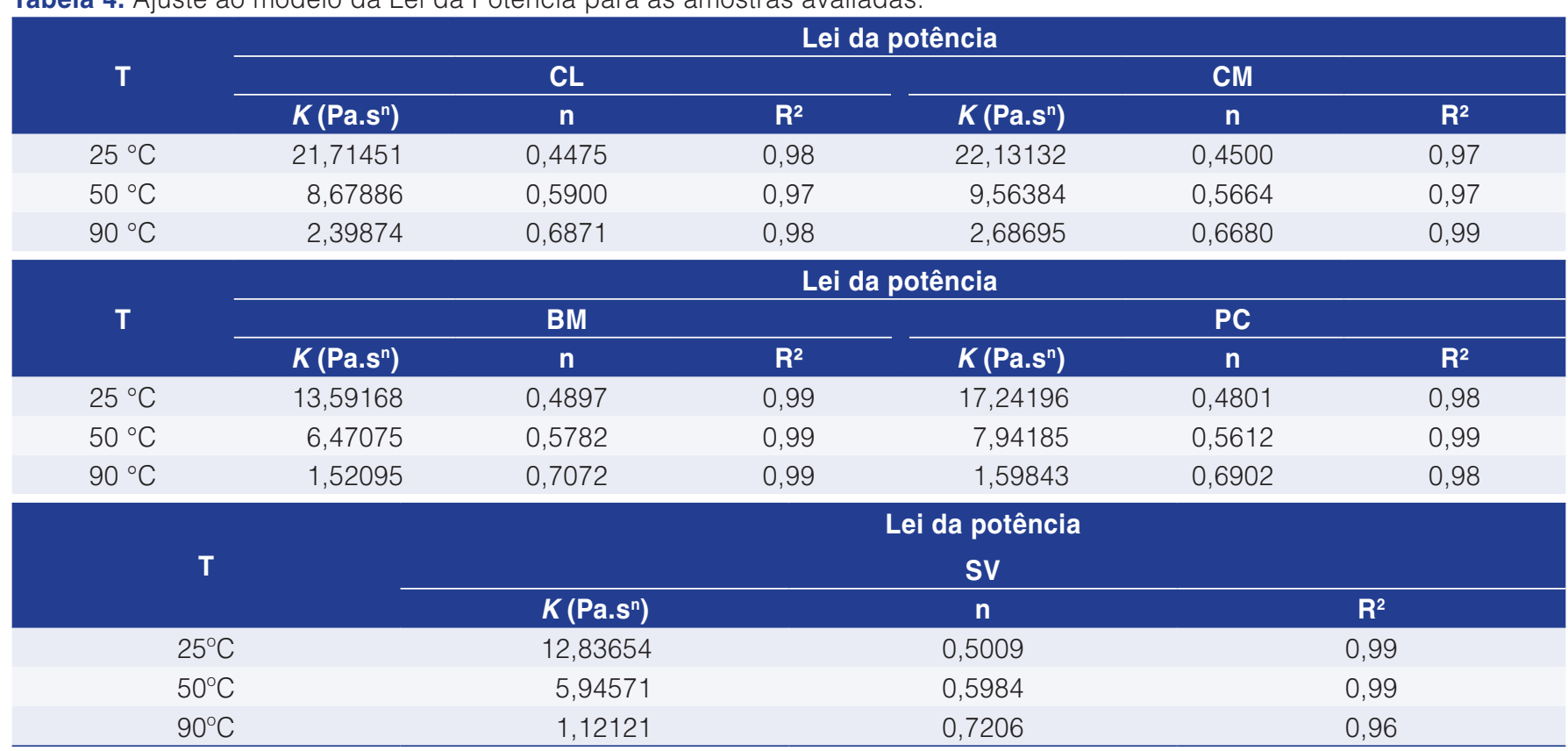

$K=$ índice de consistência; $\mathrm{n}=$ índice de comportamento.

consistência $(K)$, bem como no aumento do índice de comportamento (n). O mesmo comportamento foi observado por Zainal et al. (2000) na avaliação do efeito da temperatura (65 a $85^{\circ} \mathrm{C}$ ) no índice de consistência e no índice de comportamento de suco de goiaba ( 9 a $11^{\circ}$ Brix). Esses autores verificaram uma queda exponencial do índice de consistência e uma elevação exponencial do índice de comportamento, com o aumento da temperatura.

O comportamento reológico observado está de acordo com o que foi reportado por Diamante e Umemoto (2015), na avaliação de propriedades reológicas de produtos de frutas e vegetais. Os autores copilaram e analisaram dados de mais de 30 publicações recentes sobre as propriedades reológicas de produtos à base de frutas. O modelo Herschel-Bulkley e Lei da Potência foram usados para descrever a maior parte do comportamento dos produtos com um comportamento pseudoplástico com $n<1$.

A Figura 6 apresenta os resultados da densidade das bases e preparados de frutas.

Os produtos à base de frutas são, de modo geral, compostos por água, açúcares, fibras e ácido. Desse modo, os resultados obtidos experimentalmente podem ser comparados a modelos de predição baseados na composição centesimal. Foram empregados os modelos teóricos de Peacock (1995) - Modelo 1m, Comini et al. (1974) - Modelo 2m, Martens (1980 apud Singh, 1982) Modelo 3m e Comini et al. (1974) - Modelo 4m.

Modelo 1:

$\rho=1000\left[1+B\left(\frac{B+200}{54000}\right)\right] \times\left[1-0.036\left(\frac{T-20}{160-T}\right)\right]\left(\mathrm{kg} / \mathrm{m}^{3}\right)(1 \mathrm{~m})$
Modelo 2:

$\mathrm{Cp}=1,256+2,931 \mathrm{Xw} \quad\left(\mathrm{kJ} /\left(\mathrm{kg} .{ }^{\circ} \mathrm{C}\right)\right)$

Modelo 3:

$\alpha=[0,057363 \times \mathrm{Xw}+0,000288(T+273)] \times 10^{-6} \times 3600\left(\mathrm{~m}^{2} / \mathrm{h}\right)$

Modelo 4:

$\mathrm{k}=0,26+0,33 \times \mathrm{Xw} \quad\left(\mathrm{W} /\left(\mathrm{m} .{ }^{\circ} \mathrm{C}\right)\right)$

Onde: $\mathrm{B}=$ teor de sólidos solúveis ( $\left.{ }^{\circ} \mathrm{Brix}\right) ; \mathrm{T}=$ temperatura $\left({ }^{\circ} \mathrm{C}\right) ; \mathrm{Xw}=$ umidade da amostra (na forma decimal).

A Tabela 5 apresenta a comparação dos resultados de ajustes das propriedades termofísicas aos modelos de predição encontrados na literatura (Modelos $1 \mathrm{~m}$ a 4m), onde foram observados erros relativos entre 0,7\% e 50\%. A melhor correlação dos dados experimentais com os dados preditos foi observada para a densidade (erro relativo entre $0,2 \%$ e $1,7 \%)$.

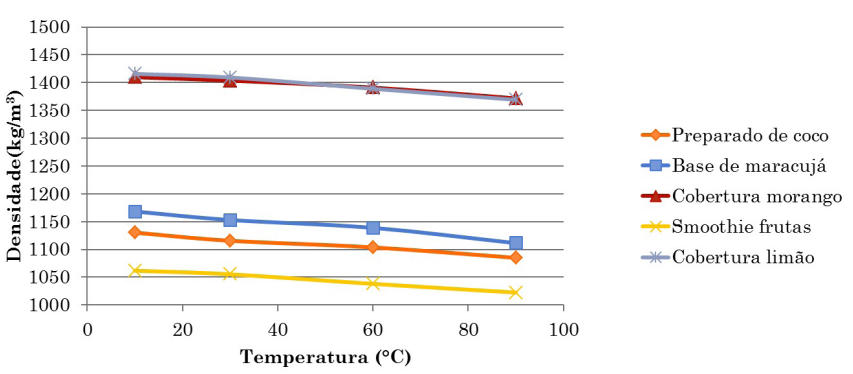

Figura 6. Representação dos resultados experimentais de densidade dos produtos à base de frutas, em função da temperatura. 
Propriedades físicas e reológicas de produtos à base de frutas

Moura, S. C. S. R. et al.

Tabela 5. Valores experimentais e teóricos das propriedades termofísicas, densidade, calor específico, difusividade térmica e condutividade térmica.

\begin{tabular}{|c|c|c|c|c|}
\hline Amostra & ${ }^{\circ}$ Brix & $\rho_{\text {experimental }}\left(\mathbf{k g} / \mathbf{m}^{3}\right)$ & $\rho_{\text {Modelo } 1}\left(\mathrm{~kg} / \mathrm{m}^{3}\right)$ & Erro (\%) \\
\hline $\mathrm{CL}$ & 83,3 & $1409,24 \pm 1,39$ & 1433,04 & 1,69 \\
\hline CM & 81,1 & $1403,63 \pm 1,09$ & 1418,23 & 1,04 \\
\hline BM & 36,3 & $1153,04 \pm 1,48$ & 1155,64 & 0,23 \\
\hline PC & 29,4 & $1125,56 \pm 1,49$ & 1121,78 & $-0,29$ \\
\hline SV & 14,5 & $1050,23 \pm 1,59$ & 1054,67 & 0,44 \\
\hline Amostra & $\mathbf{X w}$ & $\mathrm{Cp}_{\text {experimental }}\left(\mathrm{kJ} /\left(\mathbf{k g} \cdot{ }^{\circ} \mathrm{C}\right)\right)$ & $C p_{\text {Modelo 2 }}\left(\mathbf{k J} /\left(\mathbf{k g} \cdot{ }^{\circ} \mathrm{C}\right)\right)$ & Erro (\%) \\
\hline $\mathrm{CL}$ & 0,167 & $2,54 \pm 0,12$ & 1,81 & $-28,74$ \\
\hline $\mathrm{CM}$ & 0,189 & $2,21 \pm 0,11$ & 3,12 & 41,31 \\
\hline BM & 0,637 & $3,03 \pm 0,05$ & 3,32 & 9,75 \\
\hline PC & 0,706 & $3,22 \pm 0,11$ & 3,76 & 16,83 \\
\hline SV & 0,855 & $3,99 \pm 0,05$ & 4,18 & 4,94 \\
\hline Amostra & $\mathbf{X w}_{w}$ & $\alpha_{\text {experimental }}\left(m^{2} / h\right) 10^{-4}$ & $\alpha_{\text {Modelo 3 }}\left(\mathrm{m}^{2} / \mathrm{h}\right) 1^{-4}$ & Erro (\%) \\
\hline $\mathrm{CL}$ & 0,167 & $6,336 \pm 0,68$ & 3,43 & $-45,79$ \\
\hline CM & 0,189 & $6,516 \pm 0,90$ & 3,48 & $-46,59$ \\
\hline BM & 0,637 & $4,356 \pm 0,18$ & 4,40 & 1,13 \\
\hline PC & 0,706 & $4,428 \pm 0,29$ & 4,55 & 2,70 \\
\hline SV & 0,855 & $4,644 \pm 0,14$ & 4,85 & 4,55 \\
\hline Amostra & $\mathbf{X w}$ & $\mathrm{k}_{\text {experimental }}\left(\mathrm{W} /\left(\mathrm{m} .{ }^{\circ} \mathrm{C}\right)\right)$ & $\mathbf{k}_{\text {Modelo } 4}\left(\mathrm{~W} /\left(\mathrm{m} .{ }^{\circ} \mathrm{C}\right)\right)$ & Erro (\%) \\
\hline $\mathrm{CL}$ & 0,167 & $0,63 \pm 0,19$ & 0,31 & $-50,22$ \\
\hline CM & 0,189 & $0,56 \pm 0,25$ & 0,32 & $-42,74$ \\
\hline BM & 0,637 & $0,43 \pm 0,05$ & 0,47 & 9,86 \\
\hline PC & 0,706 & $0,45 \pm 0,08$ & 0,49 & 8,35 \\
\hline SV & 0,855 & $0,55 \pm 0,04$ & 0,54 & $-0,71$ \\
\hline
\end{tabular}

Errorelativo $(\%)=\left(\frac{\text { valor teórico }- \text { valor experimental }}{\text { valor experimental }}\right) \times 100$

Os resultados experimentais de densidade e difusividade são semelhantes aos apresentados por Simões (1997); sendo eles de 4,61 a 5,08 $\mathrm{m}^{2} /\left(\mathrm{h} .10^{4}\right)$ para difusividade e de 1100 a $1040 \mathrm{~kg} / \mathrm{m}^{3}$ para densidade, para polpa de manga concentrada (30 ${ }^{\circ}$ Brix) e integral (12,7 ${ }^{\circ}$ Brix), respectivamente.

Os valores experimentais obtidos de calor específico estão compatíveis com os apresentados por Rahman (2009), nos quais foram encontrados valores entre 1,78 e $3,56 \mathrm{~kJ} /\left(\mathrm{kg} .{ }^{\circ} \mathrm{C}\right)$ para polpas e frutas com diferentes concentrações (Xw 0,13 a 0,85), respectivamente.

Os valores experimentais obtidos de condutividade térmica estão dentro da faixa obtida por Greiby et al. (2014), nos quais foram encontrados valores entre 0,28 e $0,56 \mathrm{~W} /\left(\mathrm{m} .{ }^{\circ} \mathrm{C}\right)$ para polpa de cereja com diferentes concentrações (Xw 0,75 a 0,30), respectivamente.

\section{Conclusões}

Existe grande diversidade entre as características gerais dos produtos à base de fruta, principalmente quanto à quantidade de sólidos solúveis. Todas as amostras analisadas foram classificadas como fluidos pseudoplásticos, seguindo o modelo da Lei da Potência e ajustando bem ao modelo Arrhenius. Foi possível a utilização de modelo teórico para a estimativa da densidade, com erros menores que 1,7\%, mesmo com a grande variação de sólidos solúveis das amostras. Para as demais propriedades, foi identificada a necessidade da avaliação experimental das amostras, pois os modelos teóricos estudados levam em conta somente o teor de umidade das amostras e podem gerar erros de até $50 \%$ nas estimativas.

\section{Agradecimentos}

Ao CNPq pela concessão da bolsa de iniciação científica.

\section{Referências}

BARBOSA, V. C.; GARCIA-ROJAS, E. E.; COIMBRA, J. S. R.; CIPRIANO, P. A.; OLIVEIRA, E. B.; TELIS-ROMERO, J. Thermophysical and rheological properties of dulce de leche with and without coconut flakes as a function of temperature. Food Science and Technology, Campinas, v. 33, n. 1, p. 93-98, 2013. http://dx.doi.org/10.1590/S0101-20612013005000009.

BARRET, D. M.; SOMOGYI, L.; RAMASWAMY, H. Processing fruits: science and technology. 2. ed. New York: CRC Press, 2005. $119 \mathrm{p}$. 
Propriedades físicas e reológicas de produtos à base de frutas

Moura, S. C. S. R. et al.

BRASIL FOOD TRENDS 2020. São Paulo: FIESP/ITAL, 2010. 173 p.

BUDŽAKI, S.; ŠERUGA, B. Specific heat and thermal conductivity of the Croatian Unleavened Dough. International Journal of Food Properties, Philadelphia, v. 18, n. 10, p. 2300-2311, 2015. http://dx.doi.org/10.1080/10942912.2014.971180.

COMINI, G.; DEL GUIDIC, S.; LEWIS, R. W.; ZIENKIEWICZ, O. C. Finite element solution of non-linear heat conduction problems with special reference to phase change. International Journal for Numerical Methods in Engineering, Oxford, v. 8, n. 3, p. 613-624, 1974. http://dx.doi.org/10.1002/nme.1620080314.

DIAMANTE, L.; UMEMOTO, M. Rheological properties of fruits and vegetables: a review. International Journal of Food Properties, Philadelphia, v. 18, n. 6, p. 1191-1210, 2015. http://dx.doi.org/1 $0.1080 / 10942912.2014 .898653$.

DICKERSON, R. W. An apparatus for measurements of thermal diffusivity of foods. Food Technology, Chicago, v. 5, n. 19, p. 198-204, 1965.

GREIBY, I.; MISHRA, D. K.; DOLAN, K. D. Inverse method to sequentially estimate temperature-dependent thermal conductivity of cherry pomace during nonisothermal heating. Journal of Food Engineering, Essex, v. 127, p. 16-23, 2014. http://dx.doi. org/10.1016/j.jfoodeng.2013.10.039

HILL, B.; ROGER, T.; VORHAGEN, F. W. Comparative analysis of the quantization of color spaces on the basis of the CIELAB colordifference formula. ACM Transactions on Graphics, New York, v. 16, n. 2, p. 109-154, 1997. http://dx.doi.org/10.1145/248210.248212.

HWANG, M. P.; HAYAKAWA, K. A specific heat calorimeter for foods. Journal of Food Science, Chicago, v. 2, n. 44, p. 435-448, 1979. http://dx.doi.org/10.1111/j.1365-2621.1979.tb03805.x.

INSTITUTO ADOLFO LUTZ - IAL. Normas analíticas do Instituto Adolfo Lutz: métodos químicos e físicos para análises de alimentos. 3. ed. São Paulo: IAL, 1985. 533 p. (v. 1).

PEACOCK, S. Predicting physical properties of factory juices and syrups. International Sugar Journal, United Kingdom, v. 97, n. 1162, p. 571-577, 1995.

PEREIRA, C. G.; RESENDE, J. V.; GIAROLA, T. M. O. Relationship between the thermal conductivity and rheological behavior of acerola pulp: effect of concentration and temperature. LWT - Food Science and Technology, Campinas, v. 58, n. 2, p. 446-453, 2014. http://dx.doi.org/10.1016/j.Iwt.2014.04.016.
RAHMAN, S. Food properties handbook. 2. ed. New York: CRC Press, 2009. $861 \mathrm{p}$.

RAO, M. A.; RIZVI, A. A. H. Engineering properties of foods. 2. ed. New York: Marcel Dekker, 1995. 531 p.

$\mathrm{RAO}, \mathrm{C} . \mathrm{G}$. Essentials of food process engineering. Amsterdam: BIS Publishers, 2006. p. 122-129.

ROUSTAPOUR, O. R.; GAZOR, H. R. Influence of temperature and total soluble solids on thermo-physical properties of pomegranate juice. Agriculturae Conspectus Scientificus, Zagreb, v. 78, n. 4, p. 337-342, 2013.

SHARMA, S. K.; MULVANEY, S. J.; RIZVI, S. S. H. Food process engineering: theory and laboratory experiments. New York: Wiley, 2000. p. 20-186

SIMÕES, M. R. Propriedades termofísicas de polpa de manga. 1997. 84 f. Dissertação (Mestrado em Engenharia de Alimentos)Faculdade de Engenharia de Alimentos, Universidade Estadual de Campinas, 1997.

SINGH, R. P. Thermal diffusivity in food processing. Food Technology, Chicago, v. 36, n. 2, p. 87-91, 1982.

TOLEDO, R. T. Fundamentals of food process engineering. New York: Springer, 2007. p. 122-148.

VIDAL, J. R. M. B.; SIERAKOWSKI, M. R.; HAMINIUK, C. W. I.; MASSON, M. L. Propriedades reológicas da polpa de manga (Mangifera indica L. cv. Keitt) centrifugada. Ciência e Agrotecnologia, Lavras, v. 30, n. 5, p. 955-960, 2006. http:// dx.doi.org/10.1590/S1413-70542006000500020.

WILLIAMS, S. (Ed.). Official methods of analysis of the Association of Official Analytical Chemists. 19. ed. Arlington: AOAC, 1990. 1141 p.

ZAINAL, B. S.; RAHMAN, R. A.; ARIFF, A. B.; SAARI, B. N.; ASBI, B. A. Effects of temperature on the physical properties of pink guava juice at two different concentrations. Journal of Food Engineering, Essex, v. 43, n. 1, p. 55-59, 2000. http://dx.doi. org/10.1016/S0260-8774(99)00132-6.

ZULUETA, A.; ESTEVE, M. J.; FRASQUET, I.; FRÍGOLA, A. Vitamin $C$, vitamin $A$, phenolic compounds and total antioxidant capacity of new fruit juice and skim milk mixture beverages marketed in Spain. Food Chemistry, London, v. 103, n. 4, p. 1365-1374, 2007. http://dx.doi.org/10.1016/j.foodchem.2006.10.052. 